\title{
Multiconfiguration Pair-Density Functional Theory Is as Accurate as CASPT2 for Electronic Excitation
}

Chad E. Hoyer, Soumen Ghosh, Donald G. Truhlar, and Laura Gagliardi

Department of Chemistry, Chemical Theory Center, and Minnesota Supercomputing Institute, University of Minnesota, 207 Pleasant St. SE, Minneapolis, Minnesota, 55455-0431, USA.

January 25,2016

\section{Contents}

\section{Computational Methods}

Table S2. Excitation energies with various density functionals (ftPBE, trevPBE, ftrevPBE, revPBE), and CASPT2 (IPEA = 0) for moderate active spaces. Excitation energies are in $\mathrm{eV}$

Table S3. Excitation energies with various density functionals (ftPBE, trevPBE, ftrevPBE, revPBE), and CASPT2 (IPEA = 0) for 
Computational Methods. We used CASSCF as the MCSCF method in this study. For molecules for which we report only singlet valence excitation energies, the CASSCF calculations used as a reference for both CASPT2 and MC-PDFT post-SCF calculations are state-averaged over multiple states (with equal weights, followed by multi-state CASPT2). For other molecules we report singlet and triplet excitation energies using single-state CASSCF for ground states and triplet states, but the energy of the excited singlet states were calculated by state-averaged CASSCF and multi-state CASPT2. All 23 excitations were used to compare MC-PDFT with the tPBE on-top functional to CASPT2. We also compared to the performance of MC-PDFT with trevPBE, ftPBE and ftrevPBE and to TD-KS-DFT with revPBE; these additional results do not change our conclusion, and are presented below.

Charge-transfer (CT) excitations. Charge-transfer (CT) excitations represent a great challenge for KS-DFT and are severely underestimated when there is a small spatial overlap between the densities of occupied and virtual orbitals, although nonlocal functionals with high enough amounts of Hartree-Fock exchange are found to perform well for charge transfer excitations with significant overlap between occupied and virtual orbitals. ${ }^{1,2}$ Failure of KSDFT for small-overlap charge transfer is due to the underestimation of the HOMO-LUMO gap, which is often attributed to the wrong long-range behavior of these functionals.

Although so-called long-range-corrected functionals often give better performance for smalloverlap charge transfer than local or global hybrid functionals, they do not solve the problem completely $;^{2}$ furthermore, the long-range-corrected functionals often overestimate chargetransfer excitations in large-overlap regions.

Sensitivity of MC-PDFT to the active space choice and the on-top functional. In addition to the calculations with large active spaces (Table 1 in the manuscript), we performed some exploratory calculations with minimal active spaces (Table S1) to learn how much the active space can be trimmed. In particular, the active space used, except for the aromatic hydrocarbons benzene and naphthalene, was two electrons in two orbitals, denoted $(2,2)$, where the two orbitals correspond to the HOMO and LUMO - we can call this a minimal active space for spectroscopy. For benzene and naphthalene we use $(4,4)$ and $(6,6)$. With these minimal active spaces, MUE for tPBE method increases significantly $(0.40 \mathrm{eV}$ vs 0.20 $\mathrm{eV})$. It is interesting that the MUE for CASPT2 is less sensitive to the size of the active space; this is understandable because the post-SCF step in CASPT2 improves the CASSCF density, whereas the post-SCF step in MC-PDFT does not. One possible future improvement of MC-PDFT is to include the on-top functional during orbital optimization so that the densities reflect the consequences of dynamic correlation. The results for ethylene are 
particularly sensitive to the active space size because of a delicate balance between valence and Rydberg character ${ }^{3}$ in the lowest excitation of this molecule.

The MC-PDFT results are not overly sensitive to choice of the on-top density functional. In particular the results in Table $\mathrm{S} 2$ with other on-top functionals are even slightly better on average (with an average MUE of $0.18 \mathrm{eV}$ for ftrevPBE). 
Table S1. Excitation energies (in eV) with small active spaces.

\begin{tabular}{|c|c|c|c|c|c|c|}
\hline & State labeling & $\mathrm{WF}^{a}$ & CASSCF & CASPT2 & $\mathrm{tPBE}^{b}$ & Ref. \\
\hline Acetaldehyde & ${ }^{1} \mathrm{~A}^{\prime \prime} n \rightarrow \pi^{*}$ & $\mathrm{SA}(2)-(2,2)$ & 3.99 & 3.90 & 4.06 & $4.28^{4}$ \\
\hline Acetone & ${ }^{1} \mathrm{~A}_{2} n \rightarrow \pi^{*}$ & $\mathrm{SA}(2)-(2,2)$ & 4.18 & 4.04 & 4.23 & $4.43^{4}$ \\
\hline Formaldehyde & ${ }^{1} \mathrm{~A}_{2} n \rightarrow \pi^{*}$ & $\mathrm{SA}(2)-(2,2)$ & 3.62 & 3.60 & 3.59 & $4.00^{4}$ \\
\hline Pyrazine & ${ }^{1} \mathrm{~B}_{3 \mathrm{u}} n \rightarrow \pi^{*}$ & $\mathrm{SA}(2)-(2,2)$ & 5.47 & 3.48 & 3.43 & $3.97^{5,6}$ \\
\hline Pyridazine & ${ }^{1} \mathrm{~B}_{1} n \rightarrow \pi^{*}$ & $\mathrm{SA}(2)-(2,2)$ & 4.65 & 3.48 & 3.35 & $3.60^{4}$ \\
\hline Pyridine & ${ }^{1} \mathrm{~B}_{1} n \rightarrow \pi^{*}$ & $\mathrm{SA}(2)-(2,2)$ & 5.30 & 4.90 & 4.92 & $4.74^{7,8}$ \\
\hline Pyrimidine & ${ }^{1} \mathrm{~B}_{1} n \rightarrow \pi^{*}$ & $\mathrm{SA}(2)-(2,2)$ & 5.65 & 3.74 & 3.85 & $4.18^{9}$ \\
\hline$s$-Tetrazine & ${ }^{1} \mathrm{~B}_{3 \mathrm{u}} n \rightarrow \pi^{*}$ & $\mathrm{SA}(2)-(2,2)$ & 3.86 & 1.68 & 1.85 & $2.25^{4}$ \\
\hline Ethylene & ${ }^{1} \mathrm{~B}_{1 \mathrm{u}} \pi \rightarrow \pi^{*}$ & $\mathrm{SA}(2)-(2,2)$ & 7.72 & 8.05 & 7.18 & $8.02^{10}$ \\
\hline Butadiene & ${ }^{1} \mathrm{~B}_{\mathrm{u}} \pi \rightarrow \pi^{*}$ & $\mathrm{SA}(2)-(2,2)$ & 6.44 & 6.12 & 5.00 & $6.21^{11}$ \\
\hline \multirow[t]{2}{*}{ Benzene } & ${ }^{1} \mathrm{~B}_{2 \mathrm{u}}, \pi \rightarrow \pi^{*}$ & $\mathrm{SA}(2)-(6,6)$ & 4.90 & 4.91 & 5.24 & $4.90^{12}$ \\
\hline & ${ }^{3} \mathrm{~B}_{1 \mathrm{u}}, \pi \rightarrow \pi^{*}$ & $(6,6)$ & 3.78 & 4.07 & 4.33 & $4.12^{13}$ \\
\hline \multirow[t]{2}{*}{ Napthalene } & ${ }^{1} \mathrm{~B}_{3 \mathrm{u}}, \pi \rightarrow \pi^{*}$ & $\mathrm{SA}(2)-(4,4)$ & 5.94 & 4.14 & 4.02 & $4.00^{14}$ \\
\hline & ${ }^{3} \mathrm{~B}_{2 \mathrm{u}}, \pi \rightarrow \pi^{*}$ & $(4,4)$ & 2.99 & 3.66 & 3.79 & $3.11^{13}$ \\
\hline \multirow[t]{2}{*}{ Furan } & ${ }^{1} \mathrm{~B}_{2}, \pi \rightarrow \pi^{*}$ & $\mathrm{SA}(2)-(2,2)$ & 6.72 & 6.7 & 6.4 & $6.06^{15}$ \\
\hline & ${ }^{3} \mathrm{~B}_{2}, \pi \rightarrow \pi^{*}$ & $(2,2)$ & 3.88 & 4.26 & 4.37 & $4.17^{13}$ \\
\hline \multirow[t]{2}{*}{ Hexatriene } & ${ }^{1} \mathrm{~B}_{u}, \pi \rightarrow \pi^{*}$ & $\mathrm{SA}(2)-(2,2)$ & 6.56 & 5.00 & 3.25 & $4.93^{16}$ \\
\hline & ${ }^{3} \mathrm{~B}_{\mathrm{u}}, \pi \rightarrow \pi^{*}$ & $(2,2)$ & 3.50 & 2.69 & 2.52 & $2.69^{13}$ \\
\hline \multirow[t]{2}{*}{ Water } & Singlet, $2 p_{\mathrm{x}} \rightarrow 3 s$ & $\mathrm{SA}(2)-(2,2)$ & 7.33 & 7.59 & 8.06 & $7.40^{17}$ \\
\hline & Triplet, $2 p_{\mathrm{x}} \rightarrow 3 s$ & $(2,2)$ & 6.20 & 7.29 & 7.18 & $7.00^{18}$ \\
\hline$p$ NA & $\begin{array}{l}\text { Intramolecular } \\
\mathrm{CT},{ }^{1} \mathrm{~A}_{1}, \pi \rightarrow \pi^{*}\end{array}$ & $\mathrm{SA}(2)-(2,2)$ & 5.74 & 4.74 & 4.24 & $4.30^{e}$ \\
\hline DMABN & $\begin{array}{l}\text { Intramolecular } \\
\mathrm{CT},{ }^{1} \mathrm{~A}_{1}, \pi \rightarrow \pi^{*}\end{array}$ & $\mathrm{SA}(2)-(2,2)$ & 6.61 & 4.56 & 3.79 & $4.57^{19}$ \\
\hline B-TCNE & $\begin{array}{l}\text { Intermolecular } \\
\mathrm{CT},{ }^{1} \mathrm{~A}, \pi \rightarrow \pi^{*}\end{array}$ & $\mathrm{SA}(2)-(2,2)$ & 4.20 & 3.6 & 3.68 & $3.59^{20}$ \\
\hline $\operatorname{MSE}^{c}$ valence & & & 0.53 & -0.07 & -0.24 & \\
\hline MUE $^{d}$ valence & & & 0.75 & 0.26 & 0.46 & \\
\hline $\mathrm{MSE}^{c}$ Rydberg & & & -0.44 & 0.24 & 0.42 & \\
\hline MUE $^{d}$ Rydberg & & & 0.44 & 0.24 & 0.42 & \\
\hline $\mathrm{MSE}^{c} \mathrm{CT}$ & & & 1.36 & 0.15 & -0.25 & \\
\hline $\mathrm{MUE}^{d} \mathrm{CT}$ & & & 1.36 & 0.15 & 0.31 & \\
\hline Average $f$ MSE & & & 0.48 & 0.11 & -0.02 & \\
\hline Averagef MUE & & & 0.85 & 0.22 & 0.40 & \\
\hline
\end{tabular}

$a_{(n, m)}$ specifies the active space, where $n$ is the number of electrons, $m$ is the number of orbitals, and $\mathrm{SA}(N)$ indicates that the state average is over $N$ states.

$b_{\mathrm{MC}-P D F T}$

$c_{\text {mean signed error }}$

$d_{\text {mean unsigned error }}$

$e_{\delta}$-CR-EOMCC(2,3),D/6-31+G**. 
$f_{\frac{1}{3}}$ valence $+\frac{1}{3}$ Rydberg $+\frac{1}{3}$ charge transfer

Table S2. Excitation energies with various density functionals (ftPBE, trevPBE, ftrevPBE, revPBE) and CASPT2 (IPEA $=0)$ for moderate active spaces. Excitation energies are in eV.

\begin{tabular}{|c|c|c|c|c|c|c|c|}
\hline & $\begin{array}{c}\text { State } \\
\text { labeling }\end{array}$ & $\mathrm{ftPBE}^{a}$ & trevPBE ${ }^{a}$ & ftrevPBE $^{a}$ & revPBE ${ }^{b}$ & $\begin{array}{c}\text { CASPT2 } \\
(\text { IPEA }=0.0)\end{array}$ & Ref. \\
\hline Acetaldehyde & ${ }^{1} \mathrm{~A}^{\prime \prime} n \rightarrow \pi^{*}$ & 3.88 & 3.94 & 3.89 & 4.13 & 4.14 & $4.28^{4}$ \\
\hline Acetone & ${ }^{1} \mathrm{~A}_{2} n \rightarrow \pi^{*}$ & 4.35 & 4.36 & 4.36 & 4.21 & 4.3 & $4.43^{4}$ \\
\hline Formaldehyde & ${ }^{1} \mathrm{~A}_{2} n \rightarrow \pi^{*}$ & 3.47 & 3.58 & 3.51 & 3.81 & 3.79 & $4.00^{4}$ \\
\hline Pyrazine & ${ }^{1} \mathrm{~B}_{3 \mathrm{u}} n \rightarrow \pi^{*}$ & 3.83 & 3.89 & 3.87 & 3.55 & 3.76 & $3.97^{5,6}$ \\
\hline Pyridazine & ${ }^{1} \mathrm{~B}_{1} n \rightarrow \pi^{*}$ & 3.22 & 3.27 & 3.26 & 3.15 & 3.41 & $3.60^{4}$ \\
\hline Pyridine & ${ }^{1} \mathrm{~B}_{1} n \rightarrow \pi^{*}$ & 4.66 & 4.82 & 4.66 & 4.35 & 4.84 & $4.74^{7,8}$ \\
\hline Pyrimidine & ${ }^{1} \mathrm{~B}_{1} n \rightarrow \pi^{*}$ & 4.05 & 4.08 & 4.06 & 3.78 & 4.13 & $4.18^{9}$ \\
\hline$s$-Tetrazine & ${ }^{1} \mathrm{~B}_{3 \mathrm{u}} n \rightarrow \pi^{*}$ & 2.48 & 2.50 & 2.50 & 1.87 & 2.30 & $2.25^{4}$ \\
\hline Ethylene & ${ }^{1} \mathrm{~B}_{1 \mathrm{u}} \pi \rightarrow \pi^{*}$ & 6.77 & 6.74 & 6.71 & 7.3 & 8.08 & $8.02^{10}$ \\
\hline Butadiene & ${ }^{1} \mathrm{~B}_{\mathrm{u}} \pi \rightarrow \pi^{*}$ & 6.12 & 6.01 & 6.12 & 5.39 & 6.38 & $6.21^{11}$ \\
\hline \multirow[t]{2}{*}{ Benzene } & ${ }^{1} \mathrm{~B}_{2 \mathrm{u}}, \pi \rightarrow \pi^{*}$ & 4.99 & 5.11 & 4.98 & 5.14 & 4.54 & $4.90^{12}$ \\
\hline & ${ }^{3} \mathrm{~B}_{1 \mathrm{u}}, \pi \rightarrow \pi^{*}$ & 4.14 & 4.28 & 4.13 & 3.85 & 3.75 & $4.12^{13}$ \\
\hline \multirow[t]{2}{*}{ Napthalene } & ${ }^{1} \mathrm{~B}_{3 \mathrm{u}}, \pi \rightarrow \pi^{*}$ & 4.32 & 4.42 & 4.31 & 4.03 & 3.80 & $4.00^{14}$ \\
\hline & ${ }^{3} \mathrm{~B}_{2 \mathrm{u}}, \pi \rightarrow \pi^{*}$ & 3.24 & 3.32 & 3.22 & 2.76 & 2.84 & $3.11^{13}$ \\
\hline \multirow[t]{2}{*}{ Furan } & ${ }^{1} \mathrm{~B}_{2}, \pi \rightarrow \pi^{*}$ & 6.44 & 6.53 & 6.44 & 5.84 & 6.00 & $6.06^{15}$ \\
\hline & ${ }^{3} \mathrm{~B}_{2}, \pi \rightarrow \pi^{*}$ & 4.10 & 4.22 & 4.09 & 3.86 & 3.89 & $4.17^{13}$ \\
\hline \multirow[t]{2}{*}{ Hexatriene } & ${ }^{1} \mathrm{~B}_{\mathrm{u}}, \pi \rightarrow \pi^{*}$ & 5.42 & 5.50 & 5.42 & 4.41 & 5.04 & $4.93^{16}$ \\
\hline & ${ }^{3} \mathrm{~B}_{\mathrm{u}}, \pi \rightarrow \pi^{*}$ & 2.70 & 2.75 & 2.70 & 2.23 & 2.48 & $2.69^{13}$ \\
\hline \multirow[t]{2}{*}{ Water } & Singlet, $2 p_{\mathrm{x}} \rightarrow 3 s$ & 7.43 & 7.47 & 7.42 & 6.26 & 7.56 & $7.40^{17}$ \\
\hline & Triplet, $2 p_{\mathrm{x}} \rightarrow 3 s$ & 7.08 & 7.11 & 7.07 & 5.93 & 7.14 & $7.00^{18}$ \\
\hline$p \mathrm{NA}$ & $\begin{array}{l}\text { Intramolecular } \\
\mathrm{CT},{ }^{1} \mathrm{~A}_{1}, \pi \rightarrow \pi^{*}\end{array}$ & 3.93 & 3.93 & 3.96 & 3.56 & 4.28 & $4.30^{e}$ \\
\hline DMABN & $\begin{array}{l}\text { Intramolecular } \\
\mathrm{CT},{ }^{1} \mathrm{~A}_{1}, \pi \rightarrow \pi^{*}\end{array}$ & 4.28 & 4.28 & 4.3 & 4.36 & 4.50 & $4.57^{19}$ \\
\hline B-TCNE & $\begin{array}{l}\text { Intermolecular } \\
\mathrm{CT},{ }^{1} \mathrm{~A}, \pi \rightarrow \pi^{*}\end{array}$ & 3.66 & 3.63 & 3.66 & 1.35 & 3.37 & $3.59^{20}$ \\
\hline $\begin{array}{l}\mathrm{MSE}^{c} \text { for } \\
\text { valence } \\
\text { excitation }\end{array}$ & & -0.08 & -0.02 & -0.08 & -0.33 & -0.12 & \\
\hline $\begin{array}{l}\text { MUE }^{d} \text { for } \\
\text { valence } \\
\text { excitation }\end{array}$ & & 0.27 & 0.29 & 0.26 & 0.36 & 0.18 & \\
\hline $\begin{array}{l}\mathrm{MSE}^{c} \text { Rydberg } \\
\text { excitations }\end{array}$ & & 0.06 & 0.09 & 0.05 & 1.11 & 0.15 & \\
\hline $\begin{array}{c}\text { MUE }^{d} \text { Rydberg } \\
\text { exciations }\end{array}$ & & 0.06 & 0.09 & 0.05 & 1.11 & 0.15 & \\
\hline $\mathrm{MSE}^{c} \mathrm{CT}$ & & -0.20 & -0.21 & -0.18 & -1.06 & -0.10 & \\
\hline $\mathrm{MUE}^{d} \mathrm{CT}$ & & 0.24 & 0.23 & 0.23 & 1.06 & 0.10 & \\
\hline Average $f_{\text {MSE }}$ & & -0.07 & -0.05 & -0.07 & -0.09 & -0.02 & \\
\hline Average $f_{\text {MUE }}$ & & 0.19 & 0.20 & 0.18 & 0.84 & 0.14 & \\
\hline
\end{tabular}

${ }^{a} \mathrm{MC}-\mathrm{PDFT}$

${ }^{b}$ Linear-response time-dependent Kohn-Sham density functional theory is used.

${ }^{c}$ mean signed error

${ }^{d}$ mean unsigned error

${ }^{e} \delta$-CR-EOMCC $(2,3), \mathrm{D} / 6-31+\mathrm{G}^{* *}$.

${ }^{f} \frac{1}{3}$ valence $+\frac{1}{3}$ Rydberg $+\frac{1}{3}$ charge transfer 
Table S3. Excitation energies with various density functionals (ftPBE, trevPBE, ftrevPBE, revPBE) and CASPT2 (IPEA $=0$ ) for smaller active spaces. Excitation energies are in $\mathrm{eV}$.

\begin{tabular}{|c|c|c|c|c|c|c|c|}
\hline & $\begin{array}{c}\text { State } \\
\text { Labeling }\end{array}$ & $\mathrm{ftPBE}^{a}$ & trevPBE ${ }^{a}$ & $\mathrm{ftrevPBE}^{a}$ & revPBE ${ }^{b}$ & $\begin{array}{c}\text { CASPT2 } \\
(\text { IPEA }=0.0)\end{array}$ & Ref. \\
\hline Acetaldehyde & ${ }^{1} \mathrm{~A}^{\prime \prime} n \rightarrow \pi^{*}$ & 4.03 & 4.1 & 4.05 & 4.13 & 3.61 & $4.28^{4}$ \\
\hline Acetone & ${ }^{1} \mathrm{~A}_{2} n \rightarrow \pi^{*}$ & 4.2 & 4.26 & 4.21 & 4.21 & 3.73 & $4.43^{4}$ \\
\hline Formaldehyde & ${ }^{1} \mathrm{~A}_{2} n \rightarrow \pi^{*}$ & 3.63 & 3.64 & 3.65 & 3.81 & 3.33 & $4.00^{4}$ \\
\hline Pyrazine & ${ }^{1} \mathrm{~B}_{3 \mathrm{u}} n \rightarrow \pi^{*}$ & 3.52 & 3.46 & 3.55 & 3.55 & 2.71 & $3.97^{5,6}$ \\
\hline Pyridazine & ${ }^{1} \mathrm{~B}_{1} n \rightarrow \pi^{*}$ & 3.43 & 3.38 & 3.46 & 3.15 & 2.88 & $3.60^{4}$ \\
\hline Pyridine & ${ }^{1} \mathrm{~B}_{1} n \rightarrow \pi^{*}$ & 4.86 & 4.95 & 4.88 & 4.35 & 4.45 & $4.74^{7,8}$ \\
\hline Pyrimidine & ${ }^{1} \mathrm{~B}_{1} n \rightarrow \pi^{*}$ & 3.96 & 3.88 & 3.98 & 3.78 & 3.05 & $4.18^{9}$ \\
\hline$s$-Tetrazine & ${ }^{1} \mathrm{~B}_{3 \mathrm{u}} n \rightarrow \pi^{*}$ & 1.95 & 1.87 & 1.97 & 1.87 & 0.84 & $2.25^{4}$ \\
\hline Ethylene & ${ }^{1} B_{1 u} \pi \rightarrow \pi^{*}$ & 7.16 & 7.15 & 7.1 & 7.3 & 7.86 & $8.02^{10}$ \\
\hline Butadiene & ${ }^{1} \mathrm{~B}_{\mathrm{u}} \pi \rightarrow \pi^{*}$ & 5.04 & 4.99 & 5.01 & 5.39 & 5.76 & $6.21^{11}$ \\
\hline \multirow[t]{2}{*}{ Benzene } & ${ }^{1} \mathrm{~B}_{2 \mathrm{u}}, \pi \rightarrow \pi^{*}$ & 5.15 & 5.26 & 5.14 & 5.14 & 4.56 & $4.90^{12}$ \\
\hline & ${ }^{3} B_{1 u}, \pi \rightarrow \pi^{*}$ & 4.21 & 4.35 & 4.18 & 3.85 & 3.76 & $4.12^{13}$ \\
\hline \multirow[t]{2}{*}{ Napthalene } & ${ }^{1} \mathrm{~B}_{3 \mathrm{u}}, \pi \rightarrow \pi^{*}$ & 4 & 4.03 & 4.02 & 4.03 & 3.02 & $4.00^{14}$ \\
\hline & ${ }^{3} \mathrm{~B}_{2 \mathrm{u}}, \pi \rightarrow \pi^{*}$ & 3.71 & 3.82 & 3.68 & 2.76 & 3.27 & $3.11^{13}$ \\
\hline \multirow[t]{2}{*}{ Furan } & ${ }^{1} \mathrm{~B}_{2}, \pi \rightarrow \pi^{*}$ & 6.4 & 6.36 & 6.36 & 5.84 & 6.46 & $6.06^{15}$ \\
\hline & ${ }^{3} \mathrm{~B}_{2}, \pi \rightarrow \pi^{*}$ & 4.32 & 4.39 & 4.3 & 3.86 & 4.01 & $4.17^{13}$ \\
\hline \multirow[t]{2}{*}{ Hexatriene } & ${ }^{1} \mathrm{~B}_{\mathrm{u}}, \pi \rightarrow \pi^{*}$ & 3.33 & 3.25 & 3.34 & 4.41 & 4.51 & $4.93^{16}$ \\
\hline & ${ }^{3} \mathrm{~B}_{\mathrm{u}}, \pi \rightarrow \pi^{*}$ & 2.59 & 2.54 & 2.6 & 2.23 & 2.19 & $2.69^{13}$ \\
\hline \multirow[t]{2}{*}{ Water } & Singlet, $p_{\mathrm{x}} \rightarrow 3 s$ & 8.08 & 8.07 & 8.05 & 6.26 & 7.44 & $7.40^{17}$ \\
\hline & Triplet, $2 p_{\mathrm{x}} \rightarrow 3 s$ & 7.23 & 7.21 & 7.21 & 5.93 & 7.2 & $7.00^{18}$ \\
\hline$p \mathrm{NA}$ & $\begin{array}{l}\text { Intramolecular } \\
\mathrm{CT},{ }^{1} \mathrm{~A}_{1}, \pi \rightarrow \pi^{*}\end{array}$ & 4.3 & 4.25 & 4.32 & 3.56 & 4.19 & $4.30^{e}$ \\
\hline DMABN & $\begin{array}{l}\text { Intramolecular } \\
\mathrm{CT},{ }^{1} \mathrm{~A}_{1}, \pi \rightarrow \pi^{*}\end{array}$ & 3.83 & 3.8 & 3.85 & 4.36 & 3.96 & $4.57^{19}$ \\
\hline B-TCNE & $\begin{array}{l}\text { Intermolecular } \\
\mathrm{CT},{ }^{1} \mathrm{~A}, \pi \rightarrow \pi^{*}\end{array}$ & 3.75 & 3.69 & 3.75 & 1.35 & 3.1 & $3.59^{20}$ \\
\hline $\begin{array}{l}\mathrm{MSE}^{c} \text { for valence } \\
\text { excitation }\end{array}$ & & -0.23 & -0.22 & -0.23 & -0.33 & -0.54 & \\
\hline $\begin{array}{l}\mathrm{MUE}^{d} \text { for } \\
\text { valence } \\
\text { excitation }\end{array}$ & & 0.40 & 0.45 & 0.39 & 0.36 & 0.60 & \\
\hline $\begin{array}{c}\mathrm{MSE}^{c} \text { Rydberg } \\
\text { excitations }\end{array}$ & & 0.46 & 0.44 & 0.43 & -1.11 & 0.12 & \\
\hline $\begin{array}{c}\text { MUE }^{d} \text { Rydberg } \\
\text { exciations }\end{array}$ & & 0.46 & 0.44 & 0.43 & 1.11 & 0.12 & \\
\hline $\mathrm{MSE}^{c} \mathrm{CT}$ & & -0.19 & -0.24 & -0.18 & -1.06 & -0.40 & \\
\hline $\mathrm{MUE}^{d} \mathrm{CT}$ & & 0.30 & 0.31 & 0.30 & 1.06 & 0.40 & \\
\hline Average $f_{\text {MSE }}$ & & 0.01 & -0.01 & 0.01 & -0.83 & -0.27 & \\
\hline Average $f$ MUE & & 0.39 & 0.40 & 0.37 & 0.84 & 0.37 & \\
\hline
\end{tabular}

${ }^{a} \mathrm{MC}-\mathrm{PDFT}$

${ }^{b}$ Linear-response time-dependent Kohn-Sham density functional theory is used.

${ }^{c}$ mean signed error

${ }^{d}$ mean unsigned error

${ }^{e} \delta$-CR-EOMCC(2,3),D/6-31+G**.

${ }^{f} \frac{1}{3}$ valence $+\frac{1}{3}$ Rydberg $+\frac{1}{3}$ charge transfer 


\section{References}

(1) Peach, M. J. G.; Benfield, P.; Helgaker, T.; Tozer, D. J. Excitation energies in density functional theory: An evaluation and a diagnostic test. J. Chem. Phys. 2008, 128, 044118/1-8.

(2) Li, R.; Zheng, J.; Truhlar, D. G. Density functional approximations for charge transfer excitations with intermediate spatial overlap. Phys. Chem. Chem. Phys. 2008, 12, $12697-$ 12701.

(3) Buenker, R. J., Shih, S. K., Peyerimhoff, S. D. An MRD-CI study of the vertical $1\left(\pi, \pi^{*}\right)$ $\mathrm{V}-\mathrm{N}$ transition of ethylene using an AO basis with optimized Rydderg $n \mathrm{~d} \pi$ species and two separate carbon d polarization functions. Chem. Phys. 1979, 36, 97-111.

(4) Caricato, M.; Trucks, G. W.; Frisch, M. J.; Wiberg, K. B. Electronic Transition Energies: A Study of the Performance of a Large Range of Single Reference Density Functional and Wave Function Methods on Valence and Rydberg States Compared to Experiment. $J$. Chem. Theory Comput. 2010, 6, 370-383.

(5) Walker, I. C.; Palmer, M. H. The electronic states of the azines. IV. Pyrazine, studied by VUV absorption, near-threshold electron energy-loss spectroscopy and ab initio multireference configuration interaction calculations. Chem. Phys. 1991, 153, 169-187.

(6) Weber, P.; Reimers, J. R. Ab Initio and Density Functional Calculations of the Energies of the Singlet and Triplet Valence Excited States of Pyrazine. J. Phys. Chem. A 1999, 103, 9821-9829.

(7) Walker, I. C.; Palmer, M. H.; Hopkirk, A. The electronic states of the azines. II. Pyridine, studied by VUV absorption, near-threshold electron energy loss spectroscopy and ab initio multi-reference configuration interaction calculations. Chem. Phys. 1990, 141, 365-378.

(8) Cai, Z. L.;Reimers, J. R. The Low-Lying Excited States of Pyridine. J. Phys. Chem. A 2000, 104, 8389-8408.

(9) Ferreira da Silva, F.; Almeida, D.; Martins, G.; Milosavljević, A. R.; Marinković, B. P.; Hoffmann, S. V.; Mason, N. J.; Nunes, Y.; Garcia, G.; Limão-Vieira, P. The electronic states of pyrimidine studied by VUV photoabsorption and electron energy-loss spectroscopy. Phys. Chem. Chem. Phys. 2010, 12, 6717-6731.

(10) Feller, D.; Peterson, K. A.; Davidson, E. R. A systematic approach to vertically excited states of ethylene using configuration interaction and coupled cluster techniques $J$. Chem. Phys. 2014, 141, 104302/1-21.

(11) Watson, M.; Chan, G. K. L. Excited States of Butadiene to Chemical Accuracy: Reconciling Theory and Experiment. J. Chem. Theory Comput. 2012, 8, 4013-4018.

(12) Hiraya, A.; Shobatake, K. Direct absorption spectra of jet-cooled benzene in 130-260 nm. J. Chem. Phys. 1991, 94, 7700-7706.

(13) Schreiber, M.; Silva-Junior, M. R.; Sauer, S. P. A.; Thiel, W. Benchmarks for electronically excited states: CASPT2, CC2, CCSD, and CC3. J. Chem. Phys. 2008, 128, 134110/1-25.

(14) Huebner, R. H.; Meilczarek, S. R.; Kuyatt, C. E. Electron energy-loss spectroscopy of naphthalene vapor. Chem. Phys. Lett. 1972, 16, 464-469.

(15) Flicker, W. M.; Mosher, O. A.; Kuppermann, a. Electron impact investigation of electronic excitations in furan, thiophene, and pyrrole. J. Chem. Phys. 1976, 64, 13151321.

(16) Leopold, D. G.; Pendley, R. D.; Roebber, J. L.; Hemley, R. J.; Vaida, V. Direct absorption spectroscopy of jet cooled polyenes. II. The $1^{1} B^{+} u^{\leftarrow} 1^{1} A^{-}{ }_{g}$ transitions of butadienes and hexatrienes. J. Chem. Phys. 1984, 81, 4218-4221.

(17) Mota, R.; Parafita, R.; Giuliani, A; Hubin-Franskin, M. J.; Lourenço, J. M. C.; Garcia, G.; Hoffmann, S. V.; Mason, N. J.; Ribeiro, P. A.; Raposo, M.; et al. Water VUV electronic state spectroscopy by synchrotron radiation. Chem. Phys. Lett. 2005, 416, 152159. 
(18) Chutjian, A.; Hall, R. I.; Tajmar, S. Electron-impact excitation of $\mathrm{H} 2 \mathrm{O}$ and D2O at various scattering angles and impact energies in the energy-loss range 4.2-12 ev. J. Chem. Phys. 1975, 63, 892-898.

(19) Druzhinin, S. I.; Mayer, P.; Stalke, D.; Bülow, R. V.; Noltemeyer, M.; Zachariasse, K. A. Intramolecular Charge Transfer with 1-tert-Butyl-6-cyano-1,2,3,4-tetrahydroquinoline (NTC6) and Other Aminobenzonitriles. A Comparison of Experimental Vapor Phase Spectra and Crystal Structures with Calculations. J. Am. Chem. Soc. 2010, 132, 77307744.

(20) Stein, T.; Kronik, L.; Baer, R. Reliable Prediction of Charge Transfer Excitations in Molecular Complexes Using Time-Dependent Density Functional Theory. J. Am. Chem. Soc. 2009, 131, 2818-2820. 